\title{
openheart Calcific extension towards the mitral valve causes non-rheumatic mitral stenosis in degenerative aortic stenosis: real-time 3D transoesophageal echocardiography study
}

\author{
Mai Iwataki, ${ }^{1}$ Masaaki Takeuchi, ${ }^{1}$ Kyoko Otani, ${ }^{1}$ Hiroshi Kuwaki, ${ }^{1}$ \\ Hidetoshi Yoshitani, ${ }^{1}$ Haruhiko Abe, ${ }^{2}$ Roberto M Lang, ${ }^{3}$ Robert A Levine, ${ }^{4}$ \\ Yutaka Otsuji ${ }^{1}$
}

To cite: Iwataki M,

Takeuchi M, Otani K, et al. Calcific extension towards the mitral valve causes nonrheumatic mitral stenosis in degenerative aortic stenosis: real-time 3D

transoesophageal echocardiography study. Open Heart 2014:1:e000136. doi:10.1136/openhrt-2014000136

Received 19 April 2014 Revised 14 August 2014 Accepted 25 August 2014

\section{CrossMark}

\footnotetext{
${ }^{1}$ Second Department of Internal Medicine, University of Occupational and Environmental Health, School of Medicine, Kitakyushu, Japan

${ }^{2}$ Department of Heart Rhythm Managements, University of Occupational and

Environmental Health, School of Medicine, Kitakyushu, Japan

${ }^{3}$ Noninvasive Cardiac Imaging Laboratory, University of Chicago Medical Center, Chicago, Illinois, USA

${ }^{4}$ Cardiac Ultrasound Laboratory, Massachusetts General Hospital, Boston, Massachusetts, USA
}

Correspondence to Dr Masaaki Takeuchi; takeuchi@med.uoeh-u.ac.jp

\section{ABSTRACT}

Objective: Mitral annular/leaflet calcification (MALC) is frequently observed in patients with degenerative aortic stenosis (AS). However, the impact of MALC on mitral valve function has not been established. We aimed to investigate whether MALC reduces mitral annular area and restricts leaflet opening, resulting in non-rheumatic mitral stenosis.

Methods: Real-time three-dimensional transoesophageal images of the mitral valve were acquired in 101 patients with degenerative AS and 26 control participants. The outer and inner borders of the mitral annular area (MAA) and the maximal leaflet opening angle were measured at early diastole.

The mitral valve area (MVA) was calculated as the left ventricular stroke volume divided by the velocity time integral of the transmitral flow velocity.

Results: Although the outer MAA was significantly larger in patients with AS compared to control participants $\left(8.2 \pm 1.3\right.$ vs $\left.7.3 \pm 0.9 \mathrm{~cm}^{2}, p<0.001\right)$, the inner MAA was significantly smaller $\left(4.5 \pm 1.1\right.$ vs $5.9 \pm 0.9 \mathrm{~cm}^{2}$, $p<0.001$ ), resulting in an average decrease of $45 \%$ in the effective MAA. The maximal anterior and posterior leaflet opening angle was also significantly smaller in patients with AS $\left(64 \pm 10\right.$ vs $72 \pm 8^{\circ}, p<0.001,71 \pm 12$ vs $87 \pm 7^{\circ}$, $p<0.001)$. Thus, MVA was significantly smaller in patients with AS $\left(2.5 \pm 1.0\right.$ vs $\left.3.8 \pm 0.8 \mathrm{~cm}^{2}, p<0.001\right)$. Twenty-four $(24 \%)$ patients with AS showed MVA $<1.5 \mathrm{~cm}^{2}$.

Multivariate regression analysis including parameters for mitral valve geometry revealed that a decrease in effective MAA and a reduced posterior leaflet opening angle were independent predictors for MVA.

Conclusions: Calcific extension to the mitral valve in patients with AS reduced effective MAA and the leaflet opening, resulting in a significant non-rheumatic mitral stenosis in one-fourth of the patients.

\section{INTRODUCTION}

Senile/degenerative aortic stenosis (AS) is the most common cause of valvular heart

\section{KEY MESSAGES}

What is already known about this subject?

- Senile/degenerative aortic stenosis (AS) becomes a significant medical concern in developed countries. It is frequently associated with mitral annular/leaflet calcification (MALC). Mitral annular calcification is a degenerative process and an independent risk factor of cardiovascular morbidity and mortality.

What does this study add?

- Three-dimensional (3D) transoesophageal echocardiographic assessment of mitral valve geometry revealed that MALC reduced the effective annulus area, resulting in a decreased mitral valve area in patients with severe AS Approximately one-fourth of severe patients with AS were associated with a more than moderate non-rheumatic mitral stenosis.

How might this impact on clinical practice?

- Preoperative assessment of mitral valve geometry and function in patients with severe degenerative AS using 3D transoesophageal echocardiography (3DTEE) should be routinely performed in order to determine whether concomitant mitral valve surgery will be required. 3DTEE provides useful information for optimal surgical planning and subsequent outcome in individual patients.

disease in developed countries. ${ }^{1}$ The cause of degenerative AS is the restricted aortic valve leaflet opening due to the valvular thickening or calcification, secondary to sclerosis and/or inflammation. ${ }^{2-4}$ On the other hand, rheumatic mitral stenosis (MS) has become very rare because of the rapid decline in the prevalence of rheumatic fever. ${ }^{5}$ However, the prevalence of mitral annular calcification (MAC), which is a 
Figure 1 Two-dimensional (2D) and Three-dimensional (3D) images of the mitral valve and transmitral flow profile in a patient with AS and a control participant. On the 2D image, mitral annular calcification (red arrow) was prominent in the patient with AS. The 3D zoomed image from a left atrial perspective showed a whole circumferential mitral annular calcification resembling a prosthetic mitral valve ring. In this patient with AS, the leaflet opening was also restricted (yellow arrow) and the peak transmitral flow velocity increased to $160 \mathrm{~cm} / \mathrm{s}$.
AS
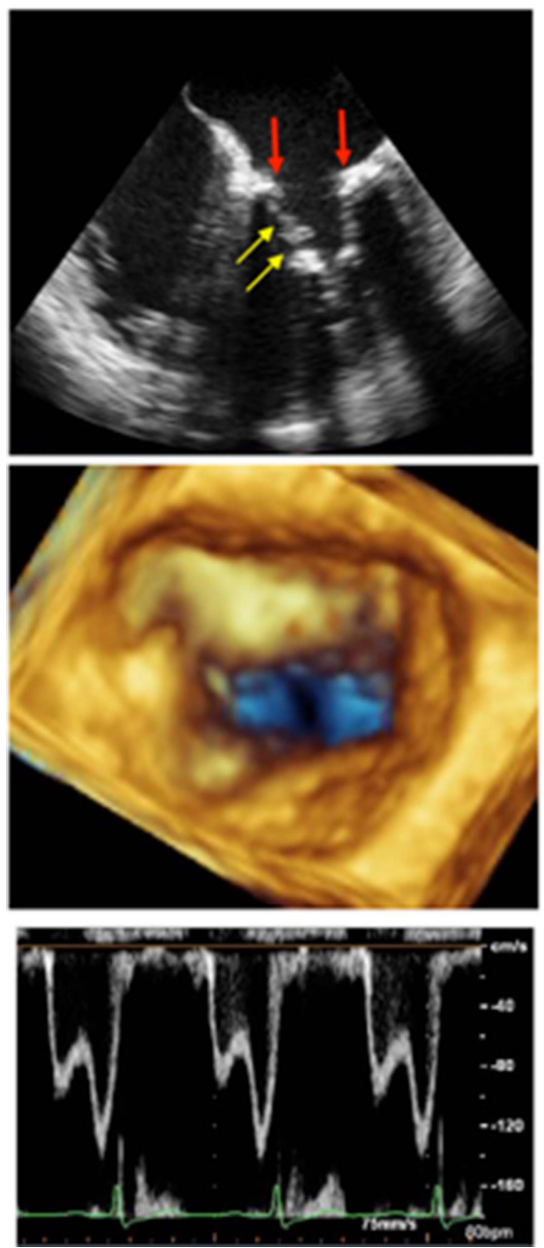

normal
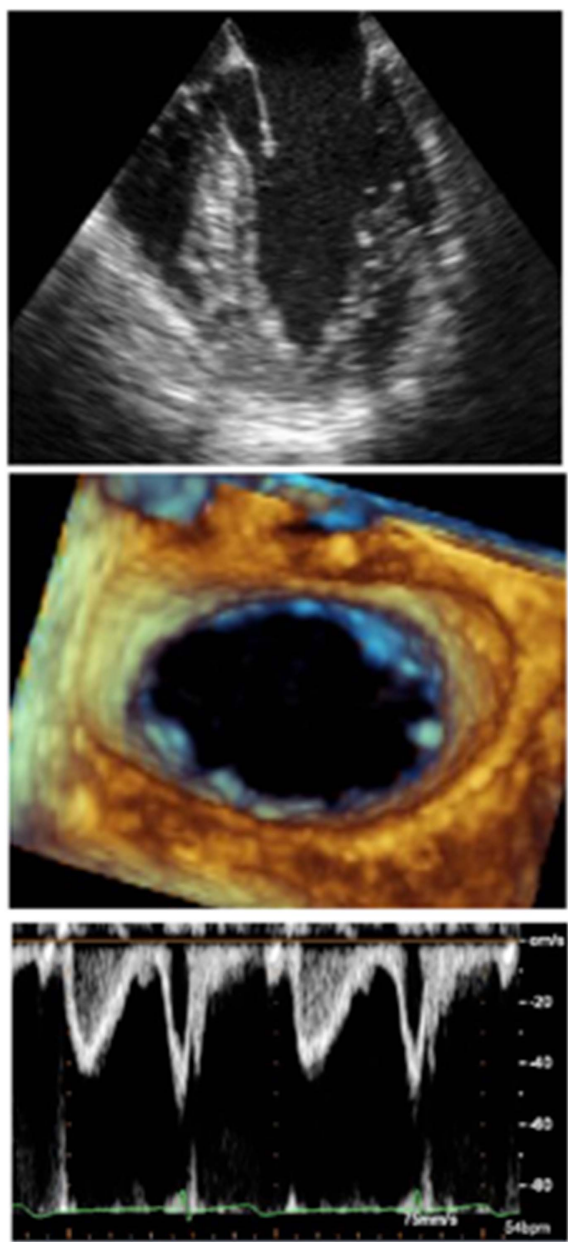

degenerative calcific process of the mitral annulus, is increasing in the ageing population. ${ }^{6}$ Although degenerative AS is known to involve both the aortic valves and systemic arteries, ${ }^{2} 37$ there have been only a few reports describing associated mitral valve apparatus pathology. ${ }^{8}$ Yong et $a l^{8}$ have described the increase in the diastolic transmitral valve pressure gradient in patients with degenerative AS. However, the underlying mechanism of this phenomenon has yet to be defined.

We have frequently observed that patients with degenerative AS have a characteristic mitral annular/leaflet calcification (MALC) with a reduction in the effective annular area as well as a reduced leaflet opening (figure 1). However, the adverse impact of this finding on mitral valve geometry and function has not been clarified. This finding raises a significant concern when operating on the aortic valve in degenerative AS, because we have to determine whether concomitant mitral valve surgery is required. We hypothesised that calcific extension into the mitral valve causes a reduction in the effective annular area and leaflet mobility, leading to non-rheumatic MS in patients with degenerative AS. Three-dimensional transoesophageal echocardiography (3DTEE) enables a quantitative evaluation of the mitral pathology ${ }^{10}$ and can potentially assess the extent and severity of MALC. Accordingly, the aims of this study were: (1) to determine the prevalence of non-rheumatic MS in patients with degenerative AS, (2) to examine the relation between the mitral valve area (MVA), mitral annulus effective area and mitral leaflet opening.

\section{METHODS}

\section{Study population}

We retrospectively enrolled 101 consecutive patients with degenerative AS who underwent 3DTEE. Degenerative AS was defined as aortic valve thickening with calcium deposition causing a reduction in aortic leaflet excursion with a reduced aortic valve area $\left(<2.0 \mathrm{~cm}^{2}\right)$ without commissural fusion. ${ }^{11}$ Since mitral valve apparatus parameters depend on the body surface area, ${ }^{12}$ we selected 26 control participants with similar body size and no aortic and mitral valve disease who underwent 3DTEE for clinical indications (ie, for sources of embolism) from our 3DTEE database. The study was approved by the institutional review board, and written informed consent was obtained for the clinical TEE procedure in all patients.

\section{DTEE measurements of mitral annular area}

3DTEE was performed by a commercially available ultrasound imaging system (iE33, Philips Healthcare, 

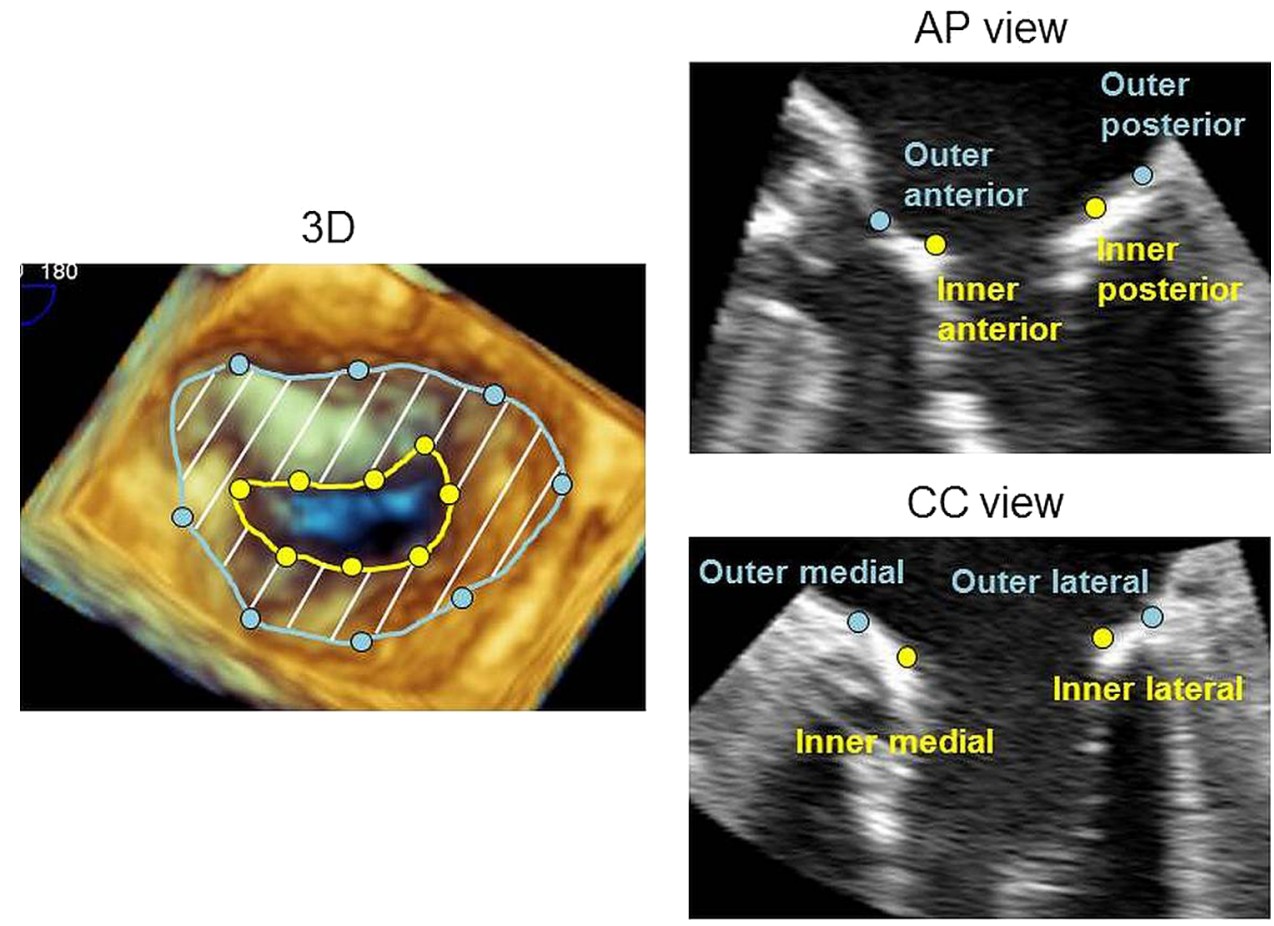

Figure 2 Three-dimensional (3D) measurement of the outer and inner/effective mitral annular area. The left panel showing a cropped 3D image of the mitral valve. From this 3D data set, an apical long-axis view of the mitral apparatus was used to determine the anterior and posterior mitral annulus, which was perpendicular to the commissure-commissure view and crossed the centre of the commissure-commissure plane (medial-lateral). The outer and inner borders of the annulus were then manually traced in multiple planes rotated around the axis connecting the centre of the mitral annulus and left ventricular apex, to measure the outer and inner/effective mitral annular area.

Andover, Massachusetts) equipped with a matrix-array transducer (X7-2t). After pharyngeal topical anaesthesia and intravenous sedation, the TEE probe was advanced into the oesophagus. Zoomed real-time 3DTEE images of the entire mitral valve were then acquired in two consecutive cardiac cycles with possibly higher volume rates (mean: $10 \pm 3 \mathrm{~Hz}$ ). 3D mitral valve analysis was performed

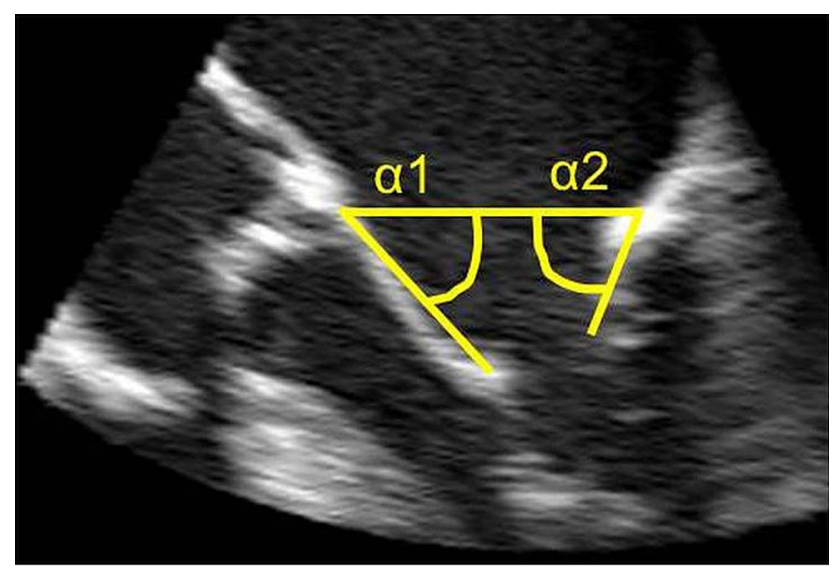

Figure 3 Measurement of the mitral leaflet opening angle. The leaflet opening angle between the anterior or posterior mitral leaflet base and annular line was measured in early diastole using a two-dimensional long-axis view of the mitral valve extracted from the three-dimensional data set. using custom software (MVQ, Phillips). After selecting an early-diastolic frame showing a maximal opening of the mitral valve leaflets, four mitral annular points, one contralateral side of the aortic valve and one nadir of the coaptation point were determined. The outer and inner borders of the annulus were then manually traced (figure 2, upper and lower panels) in multiple planes rotated around the axis perpendicular to the mitral annular plane, and the outer and inner mitral annular areas (MAA) were calculated. The calcification area was defined as outer MAA minus inner MAA.

\section{Measurements of the mitral leaflet opening angle}

The same early diastolic frame was used to measure the mitral leaflet opening angle. A two-dimensional anteroposterior view of the mitral valve, which was perpendicular to the commissure-commissure view and crossed the centre of the commissure-commissure plane (mediallateral), was extracted from the 3D data sets. The angles between the anterior or posterior mitral leaflet and inner mitral annular line $(\alpha 1 \text { and } \alpha 2)^{13-15}$ were measured using quantitative software (ImageJ, V.1.43, NIH; figure 3).

\section{Measurements of MVA}

Real-time 3DTEE images of the left ventricle (LV) were acquired using the full-volume mode (mean: $23 \pm 4 \mathrm{~Hz}$ ). Left ventricular volumes were determined using 
commercially available software (3DQ ADV, Phillips). Briefly, five anatomic landmarks (4 points for the mitral annulus and one point for the LV apex) were manually initialised on the end-diastolic frame using a nonforeshortened, apical 4-chamber and 2-chamber views were extracted from the full-volume data set. The software automatically reconstructed the 3D endocardial surface. Subsequently, the end-systolic frame was manually selected by identifying the frame showing the smallest LV cavity. Following similar initialisation, LV surface detection was repeated on this frame to calculate endsystolic volumes. Manual corrections of the endocardial border were performed when required. Finally, the computer algorithm automatically tracked the endocardial border throughout the cardiac cycle. Time domain LV volume curves were displayed, from which LV enddiastolic and end-systolic volumes and stroke volume (SV) were then calculated. ${ }^{16}$

Transmitral flow velocity was recorded using pulsed Doppler echocardiography with the sample volume placed at the tip of the mitral leaflets. Peak velocity, mean transmitral pressure gradient and velocity time integral were computed. MVA was obtained by the continuity equation $(\mathrm{SV}=\mathrm{MVA} \times$ velocity time integral of the transmitral flow). ${ }^{13}$

\section{DTEE measurements of aortic valve area}

3D zoomed images of the aortic root were acquired in two consecutive cardiac cycles (mean volume rates: 16 $\pm 5 \mathrm{~Hz}$ ). Two orthogonal long-axis views of the aortic valve and ascending aorta (anteroposterior and mediallateral projection) were obtained using the multiplanar reconstruction mode (3DQ ADV, Philips). A third plane perpendicular to both long axis planes was manipulated to obtain the orthogonal 2D cutting plane of the aortic valve. After choosing the mid-systolic frame, in which maximal aortic valve excursion was visualised, fine adjustments of the cutting plane were made to obtain the smallest aortic valve orifice. The aortic valve area was manually traced using the magnified view mode. ${ }^{17}$

\section{Statistical analysis}

Continuous data were expressed as means \pm SD. All statistical analyses were carried out using commercially available software (JMP, V.11.0, SAS Institute Inc, Cary, North Carolina, USA). Categorical variables were compared using Fisher's exact test or $\chi^{2}$ test whenever appropriate. Student's t test was used to test the differences in continuous variables between groups. Linear regression analysis was performed to investigate the relationship between the severity of MALC and other clinical characteristics. Multivariable regression analysis was used to test for independent associations between MVA and echocardiographic parameters. First, we included mitral valve geometry parameters, including outer MAA, inner/effective MAA, $\alpha 1$ and $\alpha 2$ as the independent predictors for MVA. Second, we performed multivariable analysis including clinical characteristics (age, sex, hypertension, diabetes, hypercholesterolaemia, estimated-glomerular filtration rate (eGFR) and aortic valve area) for MVA. A $p$ value of $<0.05$ was considered significant.

Table 1 Clinical characteristics of the study population

\begin{tabular}{|c|c|c|c|}
\hline & AS & Control & p Value \\
\hline Number & 101 & 26 & \\
\hline Age (year) & $76 \pm 8$ & $71 \pm 9$ & $<0.005$ \\
\hline Gender (male/female) & $51 / 50$ & $17 / 9$ & 0.17 \\
\hline $\mathrm{BSA}\left(/ \mathrm{m}^{2}\right)$ & $1.52 \pm 0.18$ & $1.59 \pm 0.17$ & 0.1 \\
\hline $\mathrm{HR}(\mathrm{bpm})$ & $74 \pm 16$ & $72 \pm 16$ & 0.59 \\
\hline $\mathrm{SBP}(\mathrm{mm} \mathrm{Hg})$ & $153 \pm 22$ & $140 \pm 22$ & $<0.05$ \\
\hline $\mathrm{DBP}(\mathrm{mm} \mathrm{Hg})$ & $78 \pm 13$ & $78 \pm 15$ & 0.96 \\
\hline \multicolumn{4}{|l|}{ Risk factors } \\
\hline Hypertension (\%) & $84(83 \%)$ & $13(50 \%)$ & $<0.001$ \\
\hline Diabetes (\%) & $32(32 \%)$ & $5(19 \%)$ & 0.21 \\
\hline Hypercholesterolaemia (\%) & $50(50 \%)$ & $9(35 \%)$ & 0.17 \\
\hline eGFR $\left(\mathrm{mL} / \mathrm{min} / 1.73 \mathrm{~m}^{2}\right)$ & $52 \pm 33$ & $77 \pm 22$ & $<0.001$ \\
\hline \multicolumn{4}{|l|}{ Left chamber volumes and EF } \\
\hline LVEDV (mL) & $88 \pm 20$ & $74 \pm 10$ & 0.001 \\
\hline LVESV $(\mathrm{mL})$ & $35 \pm 16$ & $21 \pm 7$ & $<0.001$ \\
\hline $\operatorname{LVEF}(\%)$ & $62 \pm 10$ & $72 \pm 6$ & $<0.001$ \\
\hline SV (mL) & $53 \pm 10$ & $53 \pm 5$ & 0.9 \\
\hline LA volume $(\mathrm{mL})$ & $75 \pm 23$ & $39 \pm 12$ & $<0.001$ \\
\hline \multicolumn{4}{|l|}{ Aortic valve } \\
\hline $\operatorname{AVA}\left(\mathrm{cm}^{2}\right)$ & $0.84 \pm 0.16$ & $2.58 \pm 0.53$ & $<0.001$ \\
\hline
\end{tabular}




\section{RESULTS}

The clinical characteristics of the two groups are shown in table 1.

Although the mean age was older in patients with AS compared to control participants, both groups had similar gender distribution, body surface area and heart rate. The prevalence of hypertension was significantly higher, and eGFR was significantly lower in patients with AS compared to controls. The left ventricular end-diastolic and endsystolic volumes were significantly larger, and the LV ejection fraction was significantly lower in the AS group.

\section{Mitral valve geometry}

Annular area

Outer MAA in patients with AS was significantly larger compared to control participants (table 2). In contrast, inner/effective MAA was significantly smaller in patients with AS. The lowest value of inner/effective MAA in control participants was $3.8 \mathrm{~cm}^{2}$. A total of 29 of 101 patients with AS (29\%) revealed an inner/effective MAA $<3.8 \mathrm{~cm}^{2}$. The calcification area in patients with AS was $3.7 \pm 1.1 \mathrm{~cm}^{2}$, indicating that MAC caused an average reduction of $45 \%$ (range: $26-81 \%$ ) in the effective MAA in patients with AS. Figure 4A shows scattered plots and a histogram of the calcification area in both groups. The calcification area was significantly larger in patients with AS compared to control participants with a few overlaps between the two groups.

\section{Mitral valve opening angle}

The angle between the anterior mitral leaflet and mitral annular line $(\alpha 1)$ and the angle between the posterior mitral leaflet and mitral annular line $(\alpha 2)$ at early diastole was significantly reduced in patients with AS compared to control participants.

\section{Mitral valve area}

The mean pressure gradient across the mitral valve was significantly higher and MVA was significantly smaller in patients with AS than control participants. A total of 24 patients with AS (24\%) showed MVA $<1.5 \mathrm{~cm}^{2}$, and 1 of these patients $(1 \%)$ revealed $M V A<1.0 \mathrm{~cm}^{2}$. Figure $4 \mathrm{~B}$ depicts scatter plots and a histogram of MVA in both groups. The mitral valve area in the AS group was significantly smaller than that in the control group.

Among 101 patients with AS, 63 had dyspnoea on exertion suggestive of heart failure symptoms. MVA was not different between patients with dyspnoea and those without (dyspnoea: $2.53 \pm 0.98 \mathrm{~cm}^{2}$ vs no dyspnoea: 2.44 $\left.\pm 1.02 \mathrm{~cm}^{2}\right)$. We observed a significant correlation between MVA and SV $(\mathrm{r}=0.52, \mathrm{p}<0.001)$, reflecting that the reduction of MVA is associated with the reduction of SV.

\section{Regression analysis}

A significant correlation was noted between MVA and inner MAA $(\mathrm{r}=0.59, \mathrm{p}<0.001)$. MVA was also weakly correlated with the calcification area $(\mathrm{r}=0.32, \mathrm{p}<0.005)$ and the posterior leaflet opening angles $(\alpha 2$ : $r=0.35, p<0.001)$. Moreover, the calcification area correlated with the posterior leaflet opening angles ( $\alpha 2$ : $\mathrm{r}=0.27, \mathrm{p}<0.01)$. Multivariable regression analysis including parameters for mitral valve geometry revealed that a decrease in inner MAA and reduced $\alpha 2$ were independent predictors

\begin{tabular}{|c|c|c|c|}
\hline & AS & Control & p Value \\
\hline \multicolumn{4}{|l|}{ Mitral annulus } \\
\hline Outer MAA $\left(\mathrm{cm}^{2}\right)$ & $8.2 \pm 1.3$ & $7.3 \pm 0.9$ & $<0.001$ \\
\hline CC diameter (mm) & $34.9 \pm 3.5$ & $33.6 \pm 2.5$ & 0.07 \\
\hline AP diameter (mm) & $29.3 \pm 2.9$ & $26.6 \pm 2.4$ & $<0.001$ \\
\hline CC/AP diameter & $1.20 \pm 0.15$ & $1.27 \pm 0.13$ & $<0.05$ \\
\hline Inner MAA $\left(\mathrm{cm}^{2}\right)$ & $4.5 \pm 1.1$ & $5.9 \pm 0.9$ & $<0.001$ \\
\hline CC diameter (mm) & $24.8 \pm 3.7$ & $29.7 \pm 2.4$ & $<0.001$ \\
\hline AP diameter $(\mathrm{mm})$ & $21.7 \pm 3.9$ & $24.1 \pm 2.2$ & $<0.005$ \\
\hline CC/AP diameter & $1.17 \pm 0.23$ & $1.24 \pm 0.12$ & 0.13 \\
\hline \multicolumn{4}{|c|}{ Calcification area (outer MAA-inner MAA) } \\
\hline & $3.7 \pm 1.1$ & $1.3 \pm 0.2$ & $<0.001$ \\
\hline \multicolumn{4}{|l|}{ Mitral valve } \\
\hline $\operatorname{MVA}\left(\mathrm{cm}^{2}\right)$ & $2.5 \pm 1.0$ & $3.8 \pm 0.8$ & $<0.001$ \\
\hline$M V A<1.5 \mathrm{~cm}^{2}(\%)$ & $24(24)$ & $0(0)$ & $<0.001$ \\
\hline MVA/outer MAA ratio & $0.31 \pm 0.11$ & $0.53 \pm 0.13$ & $<0.001$ \\
\hline MVA/inner MAA ratio & $0.56 \pm 0.18$ & $0.66 \pm 0.18$ & $<0.01$ \\
\hline Peak velocity $(\mathrm{m} / \mathrm{sec})$ & $1.09 \pm 0.31$ & $0.7 \pm 0.15$ & $<0.001$ \\
\hline Mean PG (mm Hg) & $1.69 \pm 1.31$ & $0.68 \pm 0.44$ & $<0.001$ \\
\hline \multicolumn{4}{|c|}{ Diastolic mitral leaflet opening angle (degree) } \\
\hline$\alpha 1$ & $64 \pm 10$ & $72 \pm 8$ & $<0.001$ \\
\hline$\alpha 2$ & $71 \pm 12$ & $87 \pm 7$ & $<0.001$ \\
\hline
\end{tabular}


Figure 4 Histogram and scatter plot of calcification area $(A)$ and mitral valve area $(B)$. Note the increased calcification area and decreased mitral valve area in patients with AS. However, the histogram shows no specific distribution pattern of calcification area and MVA in patients with AS, suggesting a single entity for these abnormalities.
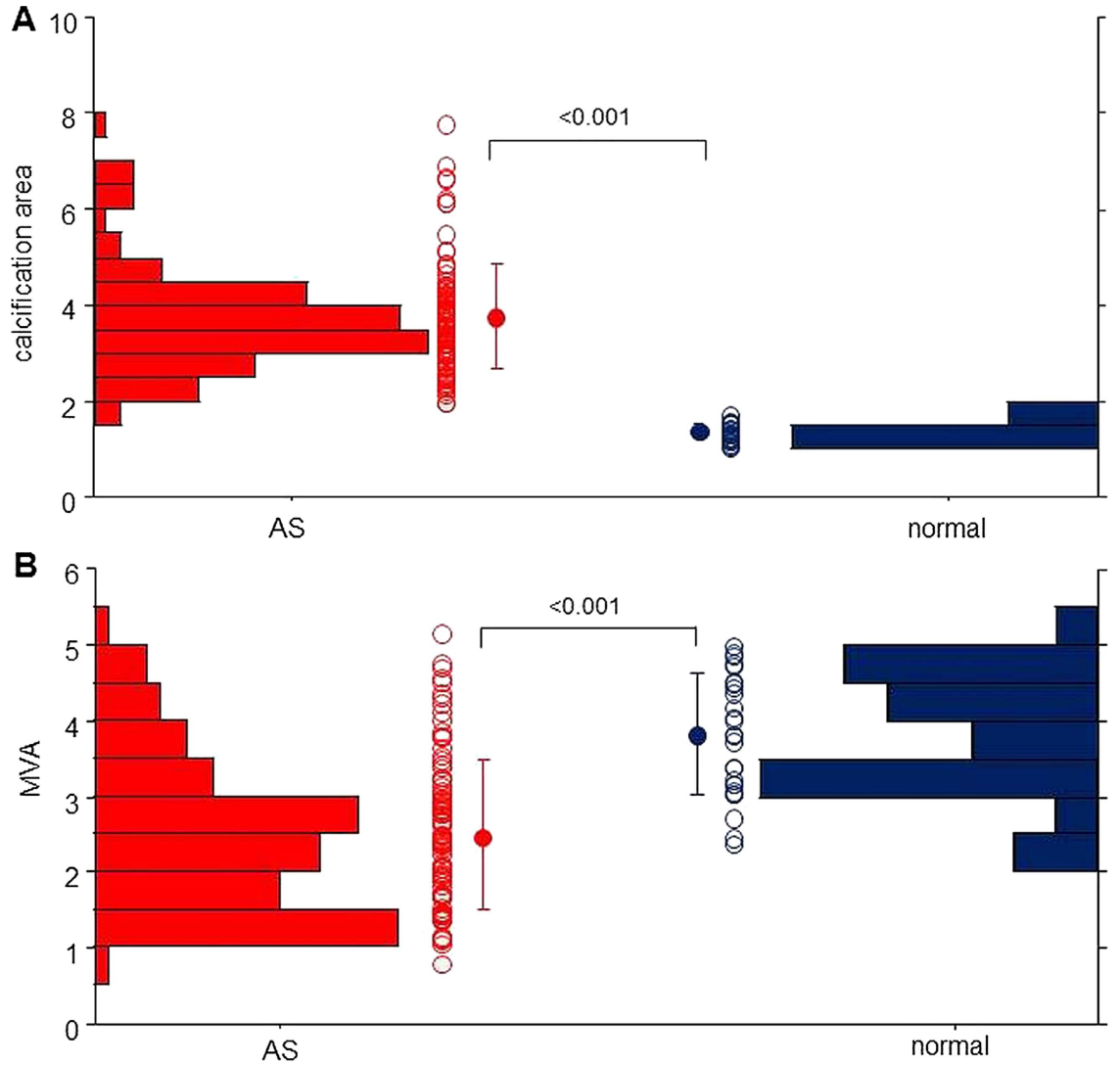

for reduced MVA. Multivariable regression analysis including clinical characteristics revealed that female gender, diabetes mellitus and aortic valve area were independent predictors for MVA (table 3).

\section{Interobserver and intraobserver variability}

In both groups, for all three parameters, the interobserver variability was below $15 \%$, while the intraobserver variability was below $10 \%$, reflecting good reproducibility, comparable to that of most clinically used echocardiography-based measurements.

\section{DISCUSSION}

\section{Previous studies}

MAC is common in the aged population. It is generally associated with mitral regurgitation, and its pathological characteristics include a semilunar deposit of calcium within the annulus fibrosis, with limited extension to the leaflet tissue. ${ }^{18}$ Although MS caused by MAC was rarely reported, Akram et $a l^{19}$ recently reported that of the 70 consecutive patients who were diagnosed with MS (mean diastolic pressure gradient $>2 \mathrm{~mm} \mathrm{Hg}$ ), $19 \%$ had non-rheumatic MS. They also reported that patients with non-rheumatic MS had larger mitral valve areas with a lower pressure gradient across the mitral valve, reflecting a milder degree of MS, compared to patients with rheumatic MS. Although the precise mechanisms for this entity remain to be determined, protruding calcification, reduction of normal mitral annular dilation during diastole and impaired anterior mitral leaflet mobility are possible mechanisms responsible for the increased mitral gradient caused by MAC. ${ }^{6}{ }^{19}$

A recent catheterisation study reported that a significant transmitral valve gradient is commonly seen in patients with severe calcific AS who underwent balloon aortic valvuloplasty. ${ }^{8}$ However, no morphological data of the mitral valve were mentioned regarding the underlying mechanism of this transmitral valve pressure

Table 3 Multivariable linear regression analysis for predicting mitral valve area

\begin{tabular}{llll}
\hline Variables & SE & $\mathbf{t}$ & $\mathbf{p}$ Value \\
\hline $\begin{array}{l}\text { Mitral valve measurements } \\
\text { Inner MAA }\end{array}$ & 0.089 & 6.028 & $<0.0001$ \\
$\quad \alpha 2$ & 0.007 & 2.747 & 0.0072 \\
$\quad \alpha 1$ & 0.008 & -1.284 & 0.2024 \\
Outer MAA & 0.079 & -0.926 & 0.3567 \\
$r^{2}=0.418$ & & & \\
Clinical characters & & & \\
Female gender & 0.095 & -3.50 & 0.0007 \\
Diabetes & 0.098 & -3.30 & 0.0014 \\
AVA & 0.618 & 2.69 & 0.0084 \\
Hypercholesterolaemia & 0.090 & 1.54 & 0.1261 \\
eGFR & 0.003 & 1.34 & 0.1824 \\
Age & 0.012 & -1.32 & 0.1911 \\
Hypertension & 0.116 & 0.71 & 0.4802 \\
$r^{2}=0.292$ & & & \\
\hline
\end{tabular}


gradient in these patients. Thus, MAC is associated with calcific aortic valve diseases, but its adverse impact on mitral valve geometry and function in patients with degenerative AS has not been established. A previous study reported anterior mitral leaflet mobility as the key determinant of a significant resting gradient across the mitral valve in patients with MAC. ${ }^{6}$

\section{Current study}

Using 3DTEE and quantitative analysis software, we demonstrated that the inner/effective MAA was significantly smaller in patients with AS, and approximately $30 \%$ of patients with AS had a smaller inner MAA than the lowest value of inner MAA in control participants. The calcification area, which reflects the severity of MAC, was also prominent in patients with AS. If we defined effective MAA as the ratio of inner MAA and outer MAA, patients with AS had on average a $45 \%$ reduction of effective MAA. Although the degree of this reduction was diverse in each patient (from 26\% to $81 \%$ ), the histogram depicting the calcification area showed no particular pattern of distribution, suggesting that there was no specific subset of patients with AS having remarkable MAC.

We also demonstrated that the maximal opening angle between the anterior mitral leaflet and inner mitral annular line was reduced in patients with AS. This suggests that calcific deposition is further extended to the body of the mitral leaflets. Multivariable regression analysis revealed that a decrease in inner MAA and a reduced posterior leaflet opening angle were independent predictors for the reduction of MVA.

In addition to the aortic valve area, multivariable regression analysis including clinical factors revealed that the female gender and the presence of diabetes were significant independent predictors for MVA. Thus, the female gender, diabetes and presence of degenerative AS itself are significant risk factors for nonrheumatic MS. Degenerative AS is considered to be a combined disease of both the aortic valve and systemic arteries. ${ }^{2}{ }^{7}$ Our results further suggest that degenerative AS is also associated with mitral valve dysfunction. This finding raises a significant concern when operating on the aortic valve in degenerative AS, because: (1) the mitral valve needs to be assessed to determine whether concomitant mitral valve surgery should be performed; (2) if required, this may pose significant comorbidities due to the surgical complexity of non-rheumatic MS. Thus, the preoperative assessment of mitral valve geometry and function in patients with degenerative AS using 3DTEE provides vital information for the optimal surgical planning and subsequent outcome.

\section{Study limitations}

This study has several limitations. First, the relatively low temporal and spatial resolution of 3D zoomed images may have influenced the measurement of the calcification area. Second, we observed clinically significant MS in $24 \%$ of patients with AS. However, its clinical implication and outcome after aortic valve replacement were not systematically evaluated. Some patients may have exaggerated the resting transmitral pressure gradients due to increases in stroke volume after aortic valve replacement. Further stress echocardiographic studies are required to determine whether reduced MVA could produce exercise-induced abnormal physiological findings in patients after aortic valve replacement. Finally, we did not have any validation data regarding 3DTEE quantification of MAA such as correlation with surgical findings, because no patients had undergone both aortic and mitral valve surgery. Further, a multimodality imaging study between 3DTEE and multidetector CT should be required to validate the accuracy of 3DTEE measurements.

\section{CONCLUSIONS}

A reduction in the effective mitral annular area by annular calcification and an impaired posterior leaflet opening were the main mechanisms for frequent nonrheumatic MS in patients with degenerative aortic valve stenosis, with significant implications for surgical planning of the aortic valve.

Contributors MI took part in data analysis and manuscript drafting. MT was involved in the conception and design of the study protocol, data analysis and manuscript drafting. KO was involved in statistical analysis and manuscript drafting. HK took part in patient selection and manuscript drafting. HY was involved in patient selection and manuscript drafting. HA, RML, RAL and YO were involved in manuscript drafting.

Funding MT was supported by a Grant-in-Aid for Scientific Research (23500574) from the Japan Society for the Promotion of Science.

\section{Competing interests None.}

\section{Patient consent Obtained.}

Provenance and peer review Not commissioned; externally peer reviewed.

Data sharing statement No additional data are available.

Open Access This is an Open Access article distributed in accordance with the Creative Commons Attribution Non Commercial (CC BY-NC 4.0) license, which permits others to distribute, remix, adapt, build upon this work noncommercially, and license their derivative works on different terms, provided the original work is properly cited and the use is non-commercial. See: http:// creativecommons.org/licenses/by-nc/4.0/

\section{REFERENCES}

1. Otto CM. Valvular aortic stenosis: disease severity and timing of intervention. J Am Coll Cardiol 2006;47:2141-51.

2. Freeman RV, Otto CM. Spectrum of calcific aortic valve disease: pathogenesis, disease progression, and treatment strategies. Circulation 2005;111:3316-26.

3. O'Brien KD. Pathogenesis of calcific aortic valve disease: a disease process comes of age (and a good deal more). Arterioscler Thromb Vasc Biol 2006;26:1721-8.

4. Otto CM. Calcific aortic stenosis: time to look more closely at the valve. N Engl J Med 2008;359:1395-8.

5. Carabello BA. Modern management of mitral stenosis. Circulation 2005;112:432-7.

6. Muddassir SM, Pressman GS. Mitral annular calcification as a cause of mitral valve gradients. Int J Cardiol 2007;123:58-62.

7. Pibarot P, Dumesnil JG. Assessment of aortic stenosis severity: check the valve but don't forget the arteries! Heart 2007;93:780-2.

8. Yong G, Ali A, Feldman T. Diastolic transmitral valve pressure gradients in patients with severe calcific aortic stenosis. Catheter Cardiovasc Interv 2009;74:957-64 
9. Chandra S, Salgo IS, Sugeng L, et al. Characterization of degenerative mitral valve disease using morphologic analysis of real-time three-dimensional echocardiographic images: objective insight into complexity and planning of mitral valve repair. Circ Cardiovasc Imaging 2011;4:24-32.

10. Sugeng L, Shernan SK, Salgo IS, et al. Live 3-dimensional transesophageal echocardiography: initial experience using the fully-sampled matrix array probe. J Am Coll Cardiol 2008;52:446-9.

11. Bonow RO, Carabello BA, Chatterjee K, et al. ACC/AHA 2006 guidelines for the management of patients with valvular heart disease: a report of the American College of Cardiology/American Heart Association task force on practice guidelines (writing committee to revise the 1998 guidelines for the management of patients with valvular heart disease): developed in collaboration with the society of cardiovascular anesthesiologists: endorsed by the society for cardiovascular angiography and interventions and the society of thoracic surgeons. Circulation 2006;114:e84-231.

12. Sonne C, Sugeng L, Watanabe N, et al. Age and body surface area dependency of mitral valve and papillary apparatus parameters: assessment by real-time three-dimensional echocardiography. Eur $J$ Echocardiogr 2009;10:287-94.

13. Kubota $\mathrm{K}$, Otsuji $\mathrm{Y}$, Ueno $\mathrm{T}$, et al. Functional mitral stenosis after surgical annuloplasty for ischemic mitral regurgitation: importance of subvalvular tethering in the mechanism and dynamic deterioration during exertion. J Thorac Cardiovasc Surg 2010;140:617-23.

14. Kuwahara $\mathrm{E}$, Otsuji $\mathrm{Y}$, Iguro $\mathrm{Y}$, et al. Mechanism of recurrent/ persistent ischemic/functional mitral regurgitation in the chronic phase after surgical annuloplasty: importance of augmented posterior leaflet tethering. Circulation 2006;114:1529-34.

15. Zhu F, Otsuji Y, Yotsumoto G, et al. Mechanism of persistent ischemic mitral regurgitation after annuloplasty: importance of augmented posterior mitral leaflet tethering. Circulation 2005;112 I396-401.

16. Meris A, Santambrogio L, Casso G, et al. Intraoperative three-dimensional versus two-dimensional echocardiography for left ventricular assessment. Anesth Analg 2014:118:711-20.

17. Nakai $\mathrm{H}$, Takeuchi $\mathrm{M}$, Yoshitani $\mathrm{H}$, et al. Pitfalls of anatomical aortic valve area measurements using two-dimensional transesophageal echocardiography and the potential of three-dimensional transesophageal echocardiography. Eur J Echocardiogr 2010;11:369-76.

18. Carpentier AF, Pellerin M, Fuzellier JF, et al. Extensive calcification of the mitral valve anulus: pathology and surgical management. $J$ Thorac Cardiovasc Surg 1996;111:718-30.

19. Akram MR, Chan T, McAuliffe $\mathrm{S}$, et al. Non-rheumatic annular mitral stenosis: prevalence and characteristics. Eur J Echocardiogr 2009;10:103-5. 